\title{
Regulation of PMP22 mRNA by G3BP1 affects cell proliferation in breast cancer cells
}

\author{
Sofia Winslow ${ }^{1}$, Karin Leandersson ${ }^{2}$ and Christer Larsson ${ }^{1 *}$
}

\begin{abstract}
Background: Regulation of mRNAs is one way to control protein levels and thereby important cellular processes such as growth, invasion and apoptosis. G3BPs constitute a family of mRNA-binding proteins, shown to be overexpressed in several cancer types, including breast, colon and pancreas cancer. G3BP has been reported to both stabilize and induce degradation of specific mRNAs.

Results: Here, we show that G3BP1, but not G3BP2, supports proliferation of several breast cancer cell lines. Global gene expression analyses of G3BP1- and G3BP2-depleted cells indicate that primarily G3BP1, and much less G3BP2, influences mRNA expression levels. Peripheral myelin protein 22 (PMP22) was one gene that was significantly influenced by G3BP1 depletion which led to a 2-3 fold increased expression. Depletion of PMP22 resulted in increased proliferation and the G3BP1-mediated effect on proliferation was not seen upon PMP22-depletion.
\end{abstract}

Conclusions: This indicates a novel role for G3BP1 in the regulation of cell proliferation in breast cancer cells, perhaps via a regulatory effect on PMP22 expression.

Keywords: G3BP, Breast cancer cells, PMP22, Proliferation, Gene expression

\section{Background}

Regulation of mRNA levels and mRNA translation is important since these processes to a large extent determine protein expression [1]. Regulation of mRNAs is frequently mediated by factors binding to AU-rich elements (ARE) in the 3'UTR [2]. Ras-GTPase activating protein SH3 domain binding proteins (G3BPs) constitute one group of such mRNA-binding proteins and consist of a family of three homologous proteins (G3BP1, G3BP2a and G3BP2b) [3]. The name originates from early reports of G3BP1 interacting with the SH3 domain of RasGAP [4], even though more recent studies have questioned this finding [5]. All G3BP proteins contain a RNA recognition motif (RRM) and have been shown to have both mRNA-stabilizing effects, exemplified by TAU mRNA [6] as well as mRNAdegrading effects, as demonstrated for $c-M Y C$ [7], BART [8], CTNNB1 [9], ATP5B [10], IGF-II, and GAS5 [11].The degrading effect has been indicated to be mediated by endonuclease activity of the G3BPs themselves [12].

\footnotetext{
* Correspondence: Christer.Larsson@med.lu.se

${ }^{1}$ Department of Laboratory Medicine, Translational Cancer Research, Lund

University, Medicon Village, Building 404:C3, Lund 223 81, Sweden

Full list of author information is available at the end of the article
}

The interactions between G3BPs and their target mRNAs have been shown to be important for regulation of processes that can influence cancer, such as cell growth and motility $[7,8]$. Overexpression of G3BP1 can promote Sphase entry in a RNA-binding domain-dependent way in fibroblasts [13] and up-regulation of G3BP1 has been detected in proliferating retinal pigment epithelial cells [14]. A potential role for G3BPs in cancer is indicated by the finding that they have been found to be expressed at high levels in many different tumor types e.g. breast $[13,15,16]$, pancreas [8], thyroid, colon, head and neck tumors [13] as well as in several cancer cell lines $[8,13]$.

The aim of this study was to investigate a putative role for G3BPs in breast cancer cell growth. G3BP1 and/or G3BP2 were depleted and the effects on cell growth and global gene expression were analyzed. We found that G3BP1 to a larger extent than G3BP2 influences mRNA expression levels and breast cancer cell proliferation. Peripheral myelin protein 22, PMP22, was one gene that was influenced by G3BP1 levels and potentially mediates the G3BP1 effect on cell proliferation.

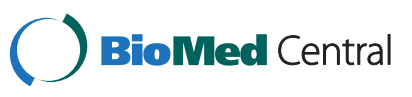

(c) 2013 Winslow et al.; licensee BioMed Central Ltd. This is an open access article distributed under the terms of the Creative Commons Attribution License (http://creativecommons.org/licenses/by/2.0), which permits unrestricted use, distribution, and reproduction in any medium, provided the original work is properly cited. 


\section{Results}

\section{G3BP1 depletion decreases cell proliferation}

To elucidate the role of G3BP proteins in breast cancer cell growth, we analyzed the effects of G3BP depletion on cell proliferation and death. Significant reduction in proliferation, measured as $\left[{ }^{3} \mathrm{H}\right]$-thymidine incorporation, was detected following G3BP1 depletion in MCF-7, MDAMB-468 and BT549 cells (Figure 1A, C and D). The same tendency, but not significant, was seen in MDA-MB-231 cells (Figure 1B). Knockdown of G3BP2 did not lead to reduced proliferation. Three separate G3BP1 siRNAs all had the same effect (Figure 1E) making it unlikely that it is a consequence of off-target effects. None of three G3BP2 siRNAs had a suppressive effect on MCF-7 cell proliferation (Figure 1F). Normalization to protein content was also done. In this case siG3BP1 led to a $\left[{ }^{3} \mathrm{H}\right]$-thymidine incorporation per cellular protein of $62.2 \pm 7.9 \%$ (mean \pm SEM, $\mathrm{n}=4$ ) compared to control cells. The effect can therefore conceivably not be explained by fewer cells, but is more likely due to lower incorporation rate per cell.

In addition, the total number of viable cells was quantified after 96 hours in culture in the presence of siRNAs targeting G3BP1 and/or G3BP2 (Figure $1 \mathrm{G}$ and H). For both MCF-7 and MDA-MB-468 cells, depletion of G3BP1 + G3BP2 led to reduced number of cells. Targeting only G3BP1 led to a significant reduction of MDA-MB468 cells and a tendency to lower MCF-7 cell number. Downregulation of G3BPs in MCF-7 cells was confirmed by Western blot (Figure 1I-K). Thus, G3BP1, but not G3BP2, seems to be a proliferation-promoting factor in breast cancer cells.

\section{G3BP-depletion does not influence cell death in breast cancer cell lines}

Since G3BP1 has been reported to be a pro-survival factor [11], we evaluated if depletion of G3BPs can affect cell survival in breast cancer cells. Knockdown of G3BPs did not influence cell death, measured as Annexin V positivity, in MCF-7 (Figure 2A) or MDA-MB-468 (Figure 2B) cells. Because of the high percentage of Annexin V positive cells under control conditions, we also quantified the number of nuclei with apoptotic morphology (Figure 2C and D). The effect was the same as was seen in the Annexin $\mathrm{V}$ assay, no G3BP siRNA influenced the number of apoptotic nuclei. However, the basal rate was much lower compared with the Annexin V data (1-2\% apoptotic nuclei vs approximately 30\% Annexin V-positive cells). This is conceivably due to the fact that floating cells are included in the Annexin V assay, thereby yielding a measure of accumulated cell death. For the nuclear morphology analysis, only cells that still are attached to the dish will be analysed, thus giving a measure of the fraction of cells that are currently undergoing certain steps in the apoptotic process. Taken together, the data indicate that neither protein is important for MCF-7 or MDA-MB-468 cell survival.

\section{Gene expression profiling indicates different roles for G3BP1 and G3BP2 in breast cancer cells}

G3BPs are considered to be RNA-binding proteins. To analyze whether altered mRNA expression may be involved in the effects of G3BP knockdown seen on cell proliferation, global gene expression in G3BP1- and/or G3BP2-depleted MCF-7 breast cancer cells was analyzed with microarray. Clustering of the expression data revealed that control cells and siG3BP2-treated cells largely clustered together (Additional file 1: Figure S1). Only one control sample diverged from this pattern. Likewise, cells treated with siG3BP1 or the combination of siG3BP1 and siG3BP2 oligonucleotides co-clustered, except for one siG3BP1 sample. The pattern was also seen when the expression levels of individual genes were compared (Additional file 2: Table S1). No genes were found to be significantly altered by downregulation of G3BP2. On the other hand, downregulation of G3BP1 led to alterations in the expression of several genes. The effect of siG3BP1 was seen both when it was used as a single agent and when it was added together with siG3BP2. This suggests that G3BP1 and G3BP2 differ in their effects on mRNA expression pattern. G3BP2 only seems to have minor effects on mRNA levels.

\section{G3BP1 suppresses PMP22 mRNA expression}

To confirm that the changes observed in the microarray analysis are due to G3BP1 depletion, qPCR analyses of the individual genes that were affected (TGFBI, TNFAIP8, CABLES1, TTC12, PMP22, SCAMP3, ARHGEF2, TNFSF10) were performed following treatment with three separate siG3BP1 oligonuclotides. For one of the genes, PMP22, the pattern observed in the microarray was replicated in the qPCR analyses (Figure 3A) and was seen for all three G3BP1 siRNAs (Figure 3B). For the other genes investigated, evaluation with $\mathrm{qPCR}$ showed no consistent effect when other G3BP1 siRNAs were tested (not shown) which made us exclude them for further analysis. In contrast to siG3BP1, siG3BP2 treatment had no major effect on PMP22 mRNA levels.

To analyze whether the effect on PMP22 mRNA is seen in other breast cancer cells as well, three additional cell lines (MDA-MB-231, MDA-MB-468 and BT549) were treated with siRNAs targeting G3BP1 and/or G3BP2 (Figure 3C-E). Knockdown of G3BP1 alone or in combination with G3BP2-depletion lead to a significant increase in PMP22 mRNA levels in all cells.

Several efforts were made to analyze PMP22 protein levels. However, two separate antibodies, or treatment 


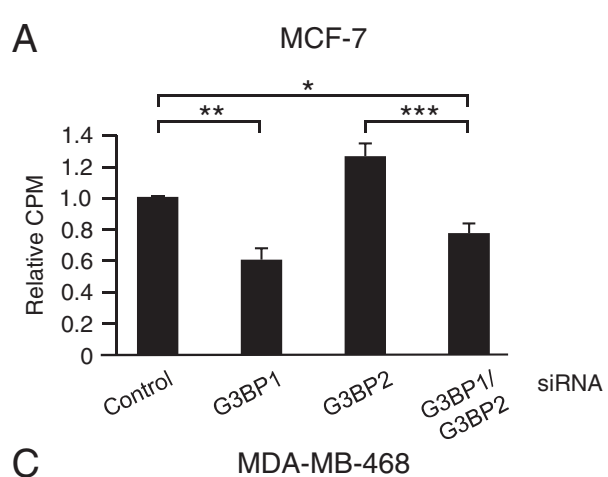

B $\quad$ MDA-MB-231

C

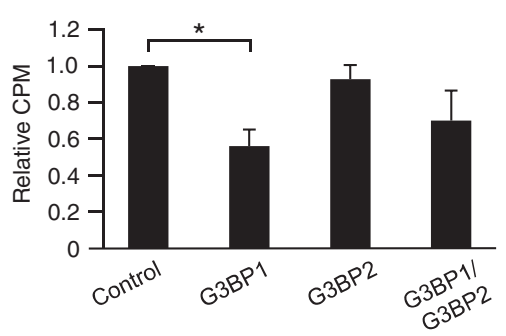

E

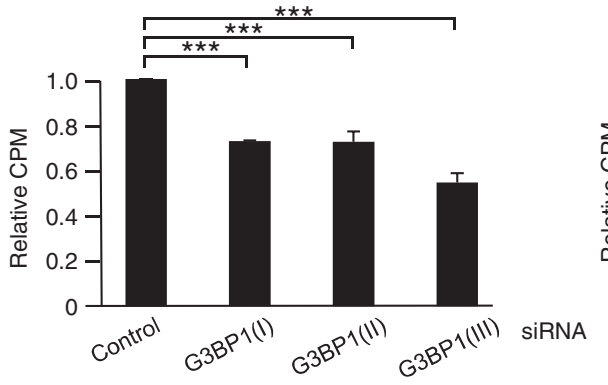

G

MCF-7

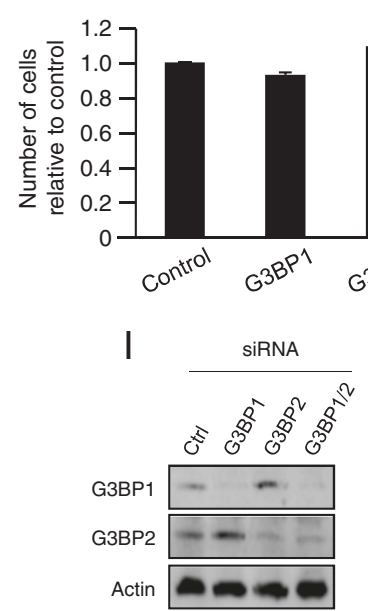

Actin

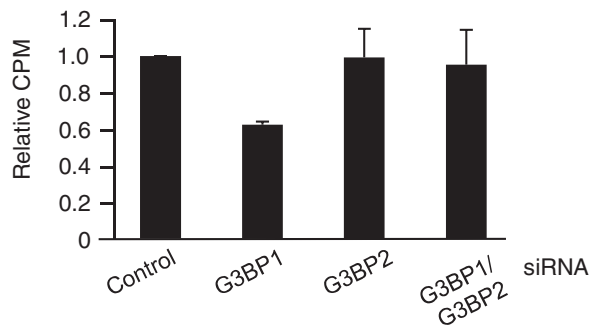

BT549

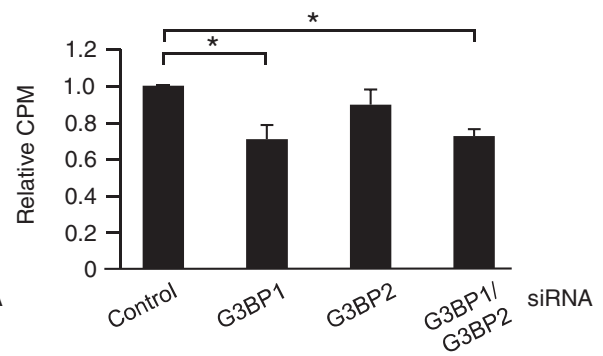

$\mathrm{F}$

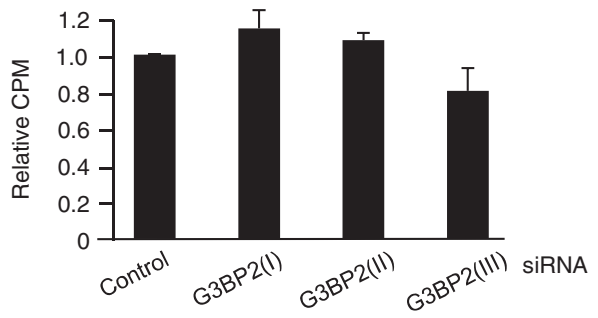

$\mathrm{H}$

MDA-MB-468

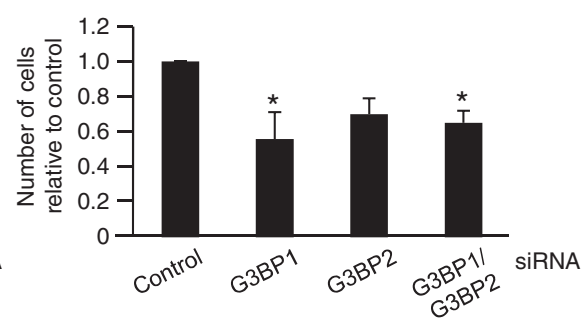

K

SiRNA
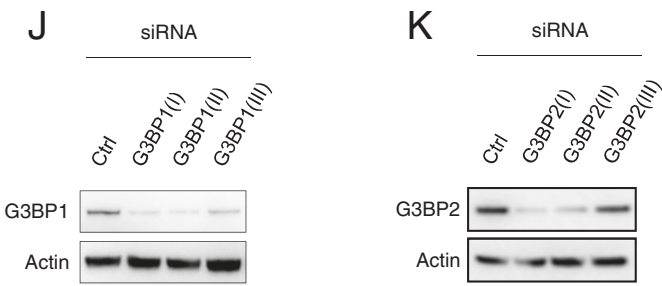

Figure 1 G3BP1 depletion decreases cell proliferation. Breast cancer cell lines MCF-7 (A, E, F), MDA-MB-231 (B), MDA-MB-468 (C) and BT549 (D) cells were transiently transfected with $40 \mathrm{nM}$ siRNA targeting G3BP1 and/or G3BP2 for 72 hours prior to $\left[{ }^{3} \mathrm{H}\right]$-thymidine incubation for 6 hours. Number of MCF-7 (G) and MDA-MB-468 (H) cells was analyzed after transfection with siRNA targeting G3BP1 and/or G3BP2 for 96 hours by counting viable cells, identified by trypan blue exclusion. Data (mean $\pm \mathrm{SEM}, \mathrm{n}=3$ ) are expressed as $\left[{ }^{3} \mathrm{H}\right]$-thymidine incorporation $(\mathbf{A}-\mathbf{F})$ and as total number of viable cells $(\mathbf{G}-\mathbf{H})$ related to control cells. ${ }^{*}<0.05,{ }^{* *}<0.01,{ }^{* * *}<0.001$ according to ANOVA followed by Duncan's multiple range test. Western blots demonstrate downregulation of G3BPs by their cognate siRNAs (I-K). 


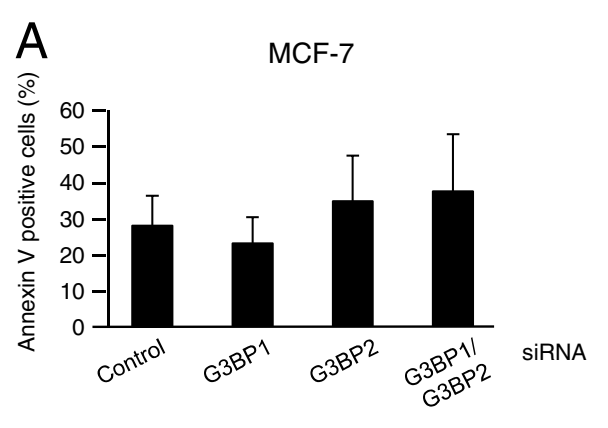

C

MCF-7

\begin{tabular}{lcc}
\hline siRNA & $\begin{array}{c}\text { Fragmented } \\
\text { nuclei (\%) }\end{array}$ & SEM \\
\hline Control & 1,17 & 0,93 \\
G3BP1 & 0,83 & 0,44 \\
G3BP2 & 1 & 0,00 \\
G3BP1/2 & 0,5 & 0,29
\end{tabular}
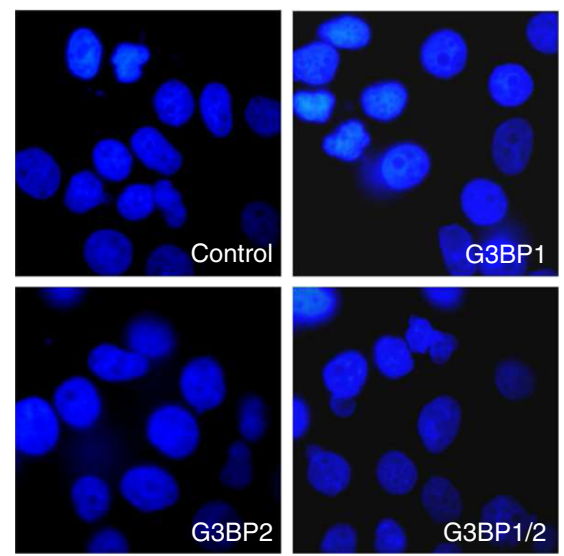

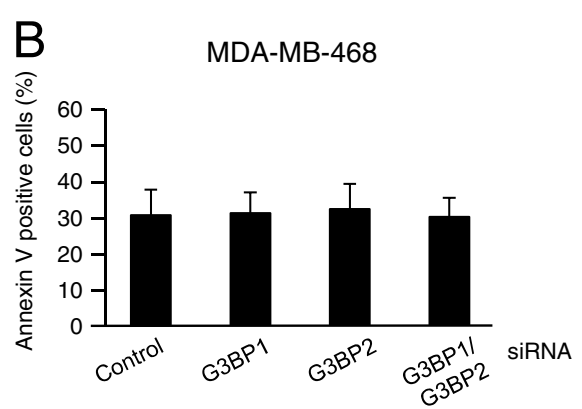

D

\begin{tabular}{lcc}
\multicolumn{3}{c}{ MDA-MB-468 } \\
\hline siRNA & $\begin{array}{c}\text { Fragmented } \\
\text { nuclei (\%) }\end{array}$ & SEM \\
\hline Control & 0,83 & 0,44 \\
G3BP1 & 0,17 & 0,17 \\
G3BP2 & 0,00 & 0,00 \\
G3BP1/2 & 0,00 & 0,00
\end{tabular}
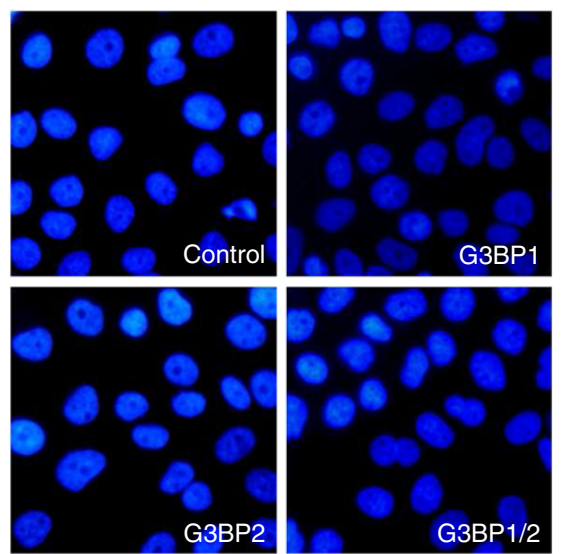

Figure 2 G3BP1 depletion does not affect cell death. Annexin V-APC staining was performed on G3BP1- and/or G3BP2-depleted MCF-7 (A) or MDA-MB-468 (B) cells, and analyzed with flow cytometry. Data (mean \pm SEM) represent the percentage of Annexin $\mathrm{V}$-positive cells in two (A) or three (B) independent experiments. Fragmented nuclei were quantified following DAPI-staining of MCF-7 (C) and MDA-MB-468 (D) cells after G3BP1 and/or G3BP2 depletion. Data are percentage fragmented nuclei (mean \pm SEM, $n=3$ ).

with PNGase F to deglycosylate the protein did not lead to detection of specific bands in Western blot analyses.

\section{Knockdown of G3BP does not influence the mRNA stability of PMP22}

Since G3BP1 is an mRNA-binding protein, a potential mechanism mediating its effect on mRNA levels could be through stabilization or destabilization of its targets [7-11]. To investigate whether this may explain its effect on PMP22 mRNA levels, G3BP-depleted MCF-7 cells were treated with actinomycin $\mathrm{D}$ for various time periods to block transcription. Calculations of the half-life of PMP22 mRNA in three independent experiments indicate no significant changes in PMP22 mRNA stability after knockdown of G3BPs (Table 1). The results suggest that G3BP1 does not suppress PMP22 mRNA levels by influencing its stability in MCF-7 cells.

\section{G3BP increases cell proliferation}

We next analyzed whether increasing the G3BP levels could further increase the proliferation rate. MCF-7 cells were transiently transfected with vectors encoding FLAG-tagged G3BP1, G3BP2a and G3BP2b. G3BP2a and G3BP2b are G3BP2 isoforms generated by alternate splicing. Overexpression of G3BP1 or G3BP2b led to significantly increased proliferation (Figure 4A). In the same line, G3BP2b overexpression induced a significant downregulation of PMP22 mRNA levels and a similar tendency could also be observed upon increased G3BP1 levels (Figure 4B). G3BP overexpression was confirmed by Western blot (Figure 4C). 


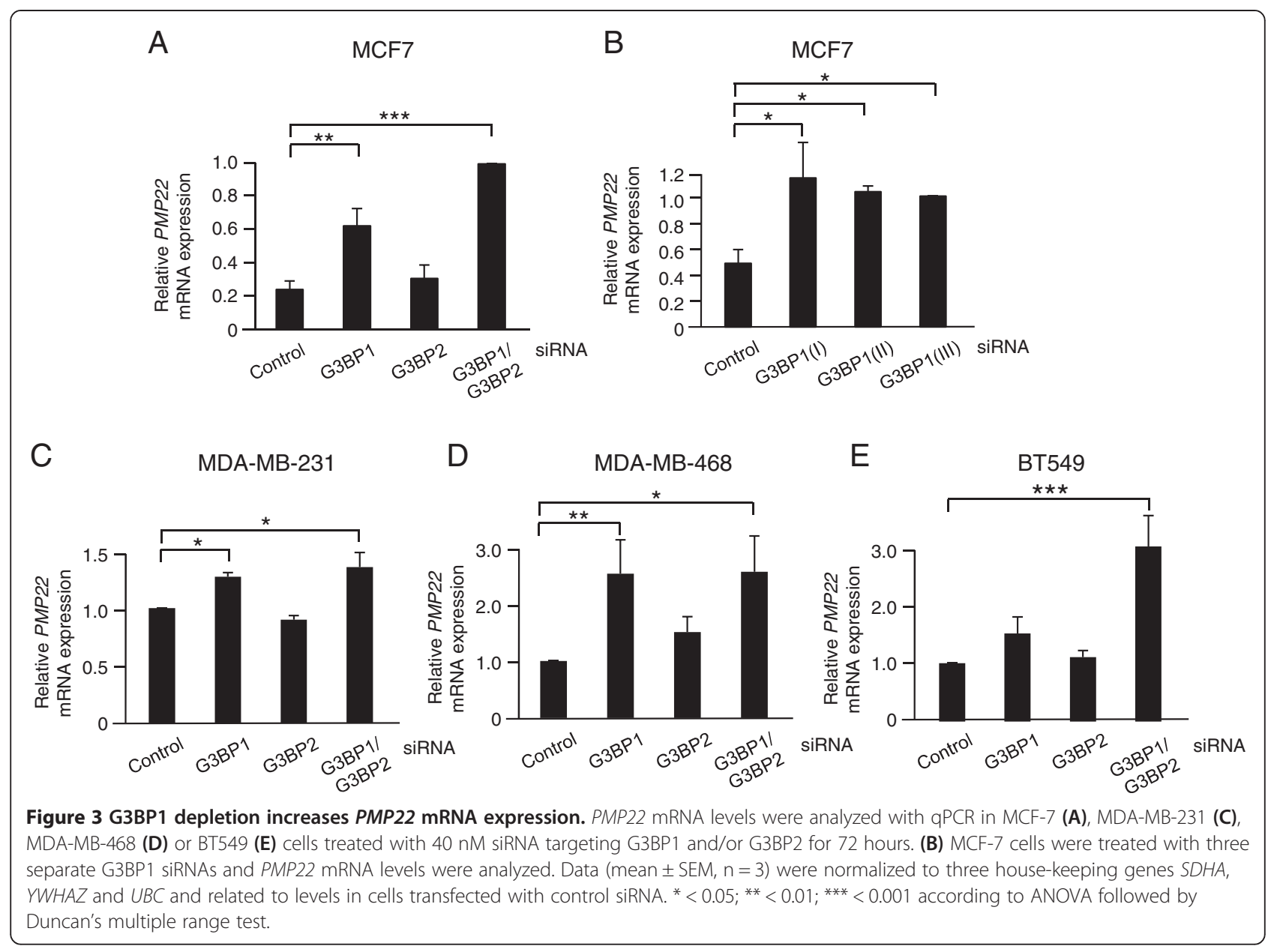

Suppression of PMP22 expression facilitates proliferation PMP22 (peripheral myelin protein 22) was first identified as a growth-arrest-specific gene (Gas3) [17,18]. It is therefore possible that the increase in PMP22 following G3BP1 downregulation, may be a mediator of the suppressed proliferation seen under this condition. PMP22 was therefore downregulated in MCF-7 cells (Figure 5A) which resulted in increased proliferation. Efficiency of PMP22 siRNA was evaluated with qPCR (Figure 5B). Concomitant downregulation of PMP22 partially reversed the decreased proliferation seen by siG3BP1 alone in MCF-7 cells (Figure 5C). In MDA-MB-231 cells no effect of siG3BP1 could be seen in the presence of siPMP22

Table 1 Determination of PMP22 mRNA stability in G3BP-depleted cells after actinomycin D treatment

\begin{tabular}{lc}
\hline SiRNA & $\begin{array}{c}\text { PMP22 } \mathbf{t}_{\mathbf{1} / \mathbf{2}} \text { (h) } \\
\text { (Mean } \pm \mathbf{S E M}, \mathbf{n}=\mathbf{3} \text { ) }\end{array}$ \\
\hline SiCtrl & $4.4 \pm 0.67$ \\
SiG3BP1 & $4.2 \pm 0.09$ \\
SiG3BP2 & $3.9 \pm 0.26$ \\
SiG3BP1/2 & $4.3 \pm 0.48$ \\
\hline
\end{tabular}

(Figure 5D). The basal PMP22 levels are substantially higher in MDA-MB-231 cells (Figure 5E) which may explain the differences between the cell lines in terms of siPMP22 effect. Together the data is in line with a hypothesis that PMP22 could be one mechanism of importance for G3BP1-mediated cell growth regulation.

\section{Discussion}

Functional studies of G3BPs have indicated a role for these RNA-binding proteins in cell growth. Here we show that G3BP1 is important for optimal breast cancer cell proliferation. This is in line with findings that downregulated levels of G3BP1 lead to suppressed growth in colon carcinoma cells [19] and that mice with G3BP1 gene deletion have decreased fetal growth and higher embryonic lethality [11]. However, we could not find any evidence for a survival role for G3BP1 as has been suggested in the same studies. In concordance with the effect on cell growth, G3BP1 $[8,13,15]$, but also G3BP2 [16] has been reported to be up-regulated in various tumor types and higher levels of G3BP1 have been reported in proliferating retinal epithelial cells [14]. We could only see effects on proliferation following G3BP1 

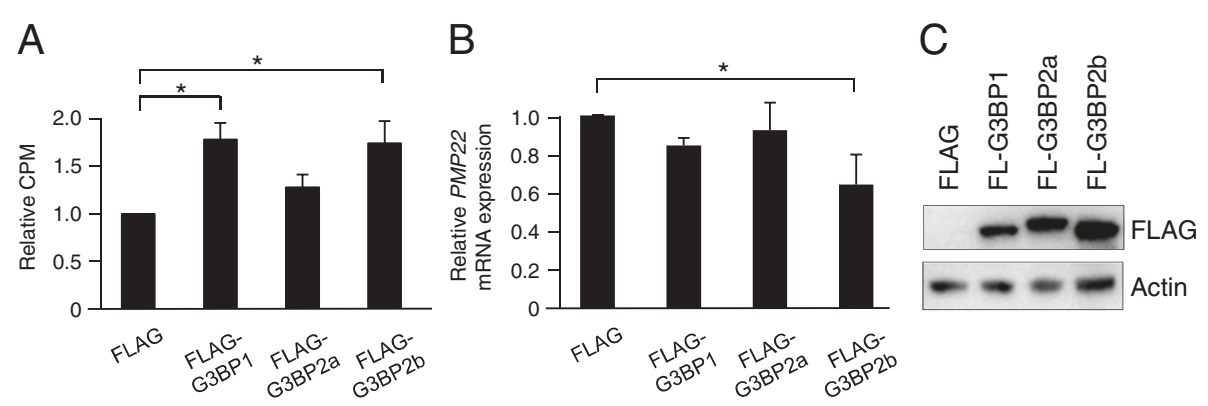

Figure 4 G3BP1 increases cell proliferation. MCF-7 cells were transiently transfected with expression vectors encoding FLAG-tagged G3BP1, G3BP2a or G3BP2b for 24 hours (A, B) prior to $\left.{ }^{3} \mathrm{H}\right]$-thymidine incubation for 6 hours or qPCR analysis. Western blot confirms G3BP overexpression (C). Data (mean $\pm \mathrm{SEM}, \mathrm{n}=3$ ) are expressed as CPM relative to control. $\mathrm{qPCR}$ data (mean $\pm \mathrm{SEM}, \mathrm{n}=3$ ) of PMP22 expression were normalized to three house-keeping genes SDHA, YWHAZ and UBC and related to control sample. ${ }^{*}<0.05$, according to ANOVA followed by Duncan's multiple range test.

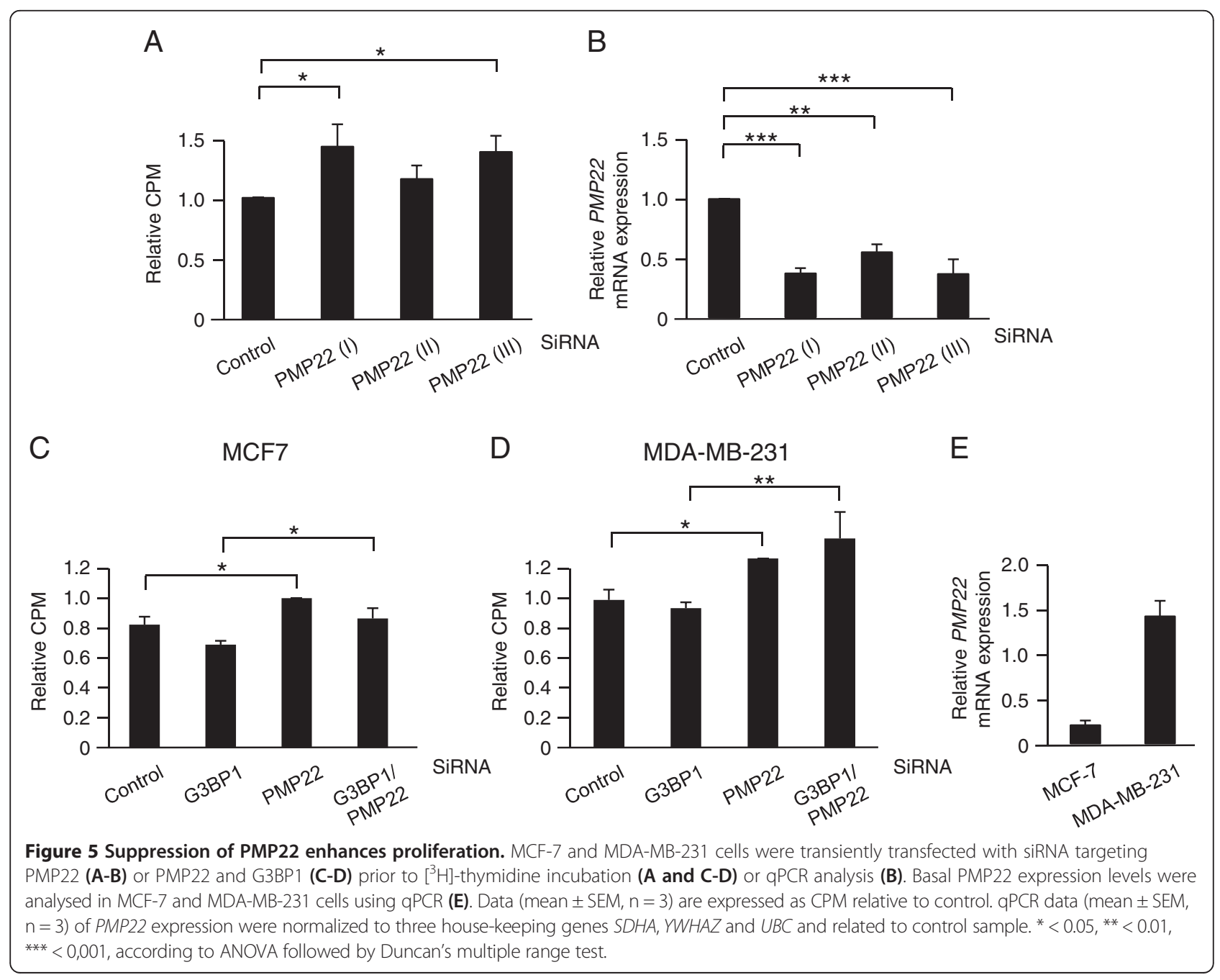


depletion and not upon G3BP2 depletion, indicating that G3BP1 and G3BP2 may have different functions in breast cancer cells.

G3BP1 and G3BP2 have around 70\% sequence similarity [20], suggesting they may have similar functions. Both G3BP1 and G3BP2 promote stress granule assembly $[21,22]$ and interact with protein kinase $C \alpha$ [23] and both G3BPs have RNA-binding motifs. However, effects on post-transcriptional regulation has this far only been reported for G3BP1 [7-12]. In most cases this results in downregulation of the target mRNAs, except for TAU which was reported to be stabilized by G3BP1 [6]. None of the reported G3BP1 targets were significantly altered in our global gene expression analysis, suggesting that the effects may depend on cellular context.

So far little is known about G3BP2 effects on gene expression. Our gene expression data indicate that G3BP1, but not G3BP2, influences mRNA levels. Thus, both proliferation and levels of mRNAs are primarily influenced by G3BP1 and not by G3BP2. There are not many other reports demonstrating differences between G3BP1 and G3BP2. However, there may be subtle differences in their subcellular localization. Although both proteins are primarily cytosolic, G3BP2 can localize to the nucleus after serum stimulation [16] whereas G3BP1 can be found in the nucleus of quiescent cells as well $[7,12]$.

The fact that G3BP1-specific effects on expression levels of mRNAs, as assayed by a global analysis, parallels effects on cell proliferation raises the hypothesis that some of the affected mRNAs may mediate the effects of G3BP1 on proliferation.

In this study we identified PMP22 mRNA levels as being influenced by G3BP1 in breast cancer cell lines. PMP22 is a $22 \mathrm{kDa}$ transmembrane glycoprotein first identified as a growth arrest specific gene (Gas3). The protein is a major component of myelin [24] and has been associated with demyelinating neuropathies [25-28]. However, there are also reports connecting PMP22 to cancer. For instance, the PMP22 gene was found to be amplified in two glioblastoma cell lines and an unusual transcript was found in the cells [29], PMP22 mRNA was higher in pancreatic adenocarcinomas than in normal tissue and was detected in cell lines [30], and high PMP22 mRNA in tumors correlated to poorer survival of breast cancer patients [31]. When it comes to cellular effects, mRNA expression has primarily been shown to be inversely correlated to cell growth [18,32-34] and overexpression of PMP22 leads to delayed G0/G1 to S-phase transition in Schwann cells [35]. Here, we show that PMP22 depletion results in increased proliferation in breast cancer cells, which supports the role of PMP22 as a growth arrest-specific gene. The effect on proliferation by PMP22 is also in line with a hypothesis that decreased PMP22 levels may mediate G3BP1-promoting effects on proliferation.
PMP22 has been reported to be post-transcriptionally regulated in the 3'UTR in Schwann cells by miRNA [36] and G3BP1 has been shown to affect mRNA levels by regulating their stability. However, our results with actinomycin D indicate no variation in stability of PMP22 mRNA in G3BP-depleted cells compared to control siRNA. The effect on PMP22 mRNA levels may therefore be due to a more indirect effect on transcriptional regulation.

\section{Conclusions}

In conclusion, our study demonstrates specific effects of G3BP1 and G3BP2 in breast cancer cells. G3BP1, but not G3BP2, supports proliferation and influences mRNA levels of different genes. We identified PMP22 as a gene that is suppressed by G3BP1 and this could be one mechanism that mediates the G3BP1 effect on proliferation.

\section{Methods}

\section{Cell culture}

MCF-7, MDA-MB-231, MDA-MB-468 and BT549 cell lines were all cultured in RPMI 1640 medium (Sigma) supplemented with $10 \%$ fetal bovine serum (EuroClone), $100 \mathrm{IU} / \mathrm{ml}$ penicillin (Gibco), and $100 \mu \mathrm{g} / \mathrm{ml}$ streptomycin (Gibco) and 1\% sodium pyruvate (Gibco). BT549 medium was additionally supplemented with $0.023 \mathrm{IU} / \mathrm{ml}$ insulin. Cells were maintained in a humidified atmosphere with $5 \% \mathrm{CO}_{2}$ at $37^{\circ} \mathrm{C}$ and seeded at a density of $1.5 \times 10^{5}$ cells/35-mm dish or $0.5 \times 10^{5}$ cells/12-well plate. For siRNA transfections, cells were seeded at a density of $1.2 \times 10^{5}$ cells/ $35-\mathrm{mm}$ dish. Actinomycin D (Sigma) was used at $4 \mu \mathrm{g} / \mathrm{ml}$ when indicated and cells from three experiments were harvested at $0,2,4$ or 8 hours. PMP22 mRNA half-times were calculated by doing a non-linear curve fit to the equation $y=a^{*} 2-x / b$ using the nls function in $R$.

\section{Plasmids and transfection}

Expression vectors encoding G3BP1, G3BP2a and G3BP2b were constructed using cDNA digested from GSTG3BP fusion vectors [23] inserted in p3xFLAG-CMV7.1 (Sigma). Cells were transfected in Optimem (Gibco) with $2 \mu \mathrm{g}$ plasmid DNA for 24 hours using Lipofectamine 2000 (Invitrogen). Transfection with $40 \mathrm{nM}$ siRNA (Stealth RNAi, Invitrogen) was performed for 72 hours (sequences are listed in Table 2).

\section{Western blot}

Cells were lysed for 15 minutes on ice with $150 \mu$ l RIPA buffer (10 mM Tris- $\mathrm{HCl} \mathrm{pH}$ 7.2, $160 \mathrm{mM} \mathrm{NaCl}, 1 \%$ Triton X-100, 1\% Na-deoxycholate, 0.1\% SDS, 1 mM EGTA and $1 \mathrm{mM}$ EDTA) supplemented with complete protease inhibitor cocktail without EDTA (Roche). Lysates were cleared by centrifugation at $14000 \times \mathrm{g}$ for $15 \mathrm{~min}$ at $4^{\circ} \mathrm{C}$ and sample buffer was added to the supernatant. Proteins 
Table 2 siRNA nucleotides

\begin{tabular}{ll}
\hline siRNA oligonucleotide & Sequence \\
\hline Control 48\% GC & UUACGGAUCGACUUAAGCCGUUGCA \\
G3BP1 | & GCGCAUUAACAGUGGUGGGAAUUA \\
G3BP1 || & CCAAGAUGAGGUCUUUGGUGGGUU \\
G3BP1 III & ACAUUUAGAGGAGCCUGUUGCUGAA \\
G3BP2 | & AACUCAUGAAGAAUUCCUUUAGCUC \\
G3BP2 I| & GCUGAAUAAAGCUCCGGAAUAUUUA \\
G3BP2 II & GGAGCCUUUGGAAGAUCCUCUCAU \\
PMP22 I & GGACACGCAACUGAUCUCUGGCAGA \\
PMP22 II & ACAUCACUGGAAUCUUCCAAAUUCU \\
PMP22 III & CCGGAGUGGCAUCUCAACUCGGAUU \\
\hline
\end{tabular}

were separated by SDS-PAGE and transferred to a PVDF membrane (Millipore). Membranes were pre-incubated with $5 \%$ dried milk in PBS followed by incubation with primary antibodies. Membranes were washed and incubated with horseradish peroxidase-labeled secondary antibody, which were detected with the SuperSignal system (Biological Industries), enhanced chemiluminescence detection system (GE Healthcare) or SuperSignal (Pierce). The chemiluminescence was captured with a chargecoupled device camera (Fujifilm). Anti-G3BP2 polyclonal antibody has been described [23]. Antibodies against G3BP1, FLAG and actin were purchased from BD Bioscience, Sigma and Santa Cruz, respectively.

\section{Proliferation assay and analysis of cell number}

For the proliferation assay, cells were seeded in triplicate at a density of $5 \times 10^{4}$ cells/well in 12-well plates and transfected with $1 \mu \mathrm{g}$ plasmid DNA or $40 \mathrm{nM}$ siRNA in $1 \mathrm{ml}$ antibiotics-free medium for 24 or 72 hours. Cells were incubated with $1 \mu \mathrm{Ci} / \mathrm{ml}[3 \mathrm{H}]$-thymidine for 6 hours before harvesting the cells with $10 \mathrm{mM}$ EDTA. The amount of radioactivity was measured with a Tri-carb 2810TR liquid scintillation analyzer (Perkin Elmer). The number of viable cells was quantified following, suspending the cells, addition of trypan blue (SigmaAldrich), and counting of trypan blue-negative cells.

\section{Annexin V-staining}

Cells were detached from the dish using trypsin and washed twice in PBS prior to Annexin V-APC staining according to supplier's protocol (BD, Pharmingen). 10000 events were acquired in channel FL-4 with a FACSCalibur (Becton Dickinson). Analyses were performed with CellQuest software (Becton Dickinson).

\section{Fragmented nuclei}

Cells were cultured on coverslips for 24 hours prior to transfection with $40 \mathrm{nM}$ siRNA according to standard procedure. After 72 hours, cells were washed with PBS and fixed with $4 \%$ paraformaldehyde in PBS for $4 \mathrm{mi}-$ nutes followed by two additional washing steps. Coverslips were mounted onto slides with VECTASHIELD mounting medium containing DAPI as counterstain (Vector Laboratories) and sealed with nail polish. For each cover slip 200 nuclei were scored for apoptotic morphology.

\section{Quantitative real-time PCR}

Total RNA was extracted from cells using RNeasy extraction kit (Qiagen). $2 \mu \mathrm{g}$ RNA was treated with DNase (Promega) followed by sample wash (Amicon Ultra, Millipore) before cDNA synthesis (High capacity cDNA RT kit, Applied Biosystems), all according to manufacturers' instructions. Quantitative PCR was performed with SYBR green master mix (Applied Biosystems) in 7300 Real-Time PCR system (Applied Biosystems), $\left(10 \mathrm{~min} 95^{\circ} \mathrm{C}, 40\right.$ cycles of $15 \mathrm{sec} 95^{\circ} \mathrm{C}$ followed by $1 \mathrm{~min} 60^{\circ} \mathrm{C}$, dissociation $60^{\circ} \mathrm{C}$ to $95^{\circ} \mathrm{C}$ ). Quantifications of expression levels were calculated by comparison of $\mathrm{Ct}$ values and normalization of the relative expression to stably expressed reference genes $S D H A, Y W H A Z$ and $U B C$. Sequences are listed in Table 3.

\section{Microarray}

Illumina DirectHyb HumanHT-12 v4.0 whole genome microarray was performed at the SCIBLU facility at Lund University. RNA was extracted from MCF-7 cells as described above, and evaluated on a Bioanalyzer 2100 (Agilent). Microarray data were background-corrected and normalized using the lumiB and lumiN functions from the lumi package in $\mathrm{R}$ [37]. Clustering was done using the AGNES (Agglomerative Hierarchical clustering algorithm) function in the cluster package. Differences in expression levels of individual genes were analyzed by fitting to a linear model followed by ranking using an empirical Bayes method (lmFit and eBayes functions in the limma package of $\mathrm{R}$ ).

Table 3 qPCR primers

\begin{tabular}{ll}
\hline Primers for qPCR & Sequence $\mathbf{5}^{\prime}$ to $\mathbf{3}^{\prime}$ \\
\hline SDHA forward & TGGGAACAAGAGGGCATCTG \\
SDHA reverse & CCACCACTGCATCAAATTCATG \\
YWHAZ forward & ACTTTGGTACATTGTGGTTCAA \\
YWHAZ reverse & CCGCCAGGACAAACCAGTAT \\
UBC forward & ATTTGGGTGCGGTTCTTG \\
UBC reverse & TGCCTTGACATTCTCGATGGT \\
PMP22 forward & ACATAGGGAAGGAGGAAGG \\
PMP22 reverse & TTGGTTGGTTGAGTTGG \\
\hline
\end{tabular}




\section{Additional files}

Additional file 1: Figure S1. Dendrogram chart of G3BP depleted MCF-7 cells. Three separate experiments of MCF-7 cells with downregulated G3BP1 (1), G3BP2 (2), G3BP1 and G3BP2 (1/2) or cells treated with control siRNA (C) were analyzed for global gene expression. The expression data were clustered with the AGNES function in the cluster package of $R$.

Additional file 2: Table S1. Differentially expressed genes. Expression data from MCF-7 cells with downregulated G3BP1 and/or G3BP2 were analyzed with the limma package of R. For each gene the fold change (Log) and adjusted p-value are shown.

\section{Abbreviations}

G3BP: Ras-GTPase activating protein SH3 domain binding proteins; PMP22: Peripheral myelin protein 22; ARE: AU-rich elements;

3'UTR: 3'untranslated region; RRM: RNA recognition motif; PNGase F: Peptide -N-Glycosidase F.

\section{Competing interests}

The authors declare that they have no competing interests.

\section{Authors' contributions}

SW carried out all the experimental work, participated in the design and assembled the drafts of the manuscript. KL participated in interpretative discussions and helped draft the manuscript. $C L$ conceived of the study, participated in the design of the experimental work, performed the statistical analyses and helped draft the manuscript. All authors read and approved the final manuscript.

\section{Acknowledgements}

This work was supported by grants from the Swedish Research Council, the Swedish Cancer Society, the Gunnar Nilsson, Ollie and Elof Ericsson, and Kock foundations, and Malmö University Hospital research funds.

\section{Author details}

'Department of Laboratory Medicine, Translational Cancer Research, Lund University, Medicon Village, Building 404:C3, Lund 223 81, Sweden.

${ }^{2}$ Department of Laboratory Medicine Malmö, Center for Molecular Pathology, Lund University, SUS Malmö, Jan Waldenströms gata 59, Malmö 205 02, Sweden.

Received: 21 May 2013 Accepted: 27 November 2013 Published: 9 December 2013

\section{References}

1. Schoenberg DR: Mechanisms of endonuclease-mediated mRNA decay. Wiley Interdiscip Rev RNA 2011, 2:582-600.

2. Wurth $\mathrm{L}$ : Versatility of RNA-Binding proteins in cancer. Comp Funct Genomics 2012, 2012:178525

3. Kennedy D, French J, Guitard E, Ru K, Tocque B, Mattick J: Characterization of G3BPs: tissue specific expression, chromosomal localisation and rasGAP(120) binding studies. J Cell Biochem 2001, 84:173-187.

4. Parker F, Maurier F, Delumeau I, Duchesne M, Faucher D, Debussche L, Dugue A, Schweighoffer F, Tocque B: A Ras-GTPase-activating protein SH3-domain-binding protein. Mol Cell Biol 1996, 16:2561-2569.

5. Annibaldi A, Dousse A, Martin S, Tazi J, Widmann C: Revisiting G3BP1 as a RasGAP binding protein: sensitization of tumor cells to chemotherapy by the RasGAP 317-326 sequence does not involve G3BP1. PLOS One 2011, 6:e29024.

6. Atlas $R$, Behar $L$, Elliott $E$, Ginzburg I: The insulin-like growth factor mRNA binding-protein IMP-1 and the Ras-regulatory protein G3BP associate with tau mRNA and HuD protein in differentiated P19 neuronal cells. J Neurochem 2004, 89:613-626.

7. Gallouzi IE, Parker F, Chebli K, Maurier F, Labourier E, Barlat I, Capony JP, Tocque B, Tazi J: A novel phosphorylation-dependent RNase activity of GAP-SH3 binding protein: a potential link between signal transduction and RNA stability. Mol Cell Biol 1998, 18:3956-3965.

8. Taniuchi K, Nishimori I, Hollingsworth MA: The N-terminal domain of G3BP enhances cell motility and invasion by posttranscriptional regulation of BART. Mol Cancer Res 2011, 9:856-866.
9. Bikkavilli RK, Malbon CC: Arginine methylation of G3BP1 in response to Wnt3a regulates beta-catenin mRNA. J Cell Sci 2011, 124:2310-2320.

10. Ortega AD, Willers IM, Sala S, Cuezva JM: Human G3BP1 interacts with beta-F1-ATPase mRNA and inhibits its translation. J Cell Sci 2010, 123:2685-2696.

11. Zekri L, Chebli K, Tourriere H, Nielsen FC, Hansen TV, Rami A, Tazi J: Control of fetal growth and neonatal survival by the RasGAP-associated endoribonuclease G3BP. Mol Cell Biol 2005, 25:8703-8716.

12. Tourriere H, Gallouzi IE, Chebli K, Capony JP, Mouaikel J, van der Geer P, Tazi J: RasGAP-associated endoribonuclease G3Bp: selective RNA degradation and phosphorylation-dependent localization. Mol Cell Biol 2001, 21:7747-7760

13. Guitard E, Parker F, Millon R, Abecassis J, Tocque B: G3BP is overexpressed in human tumors and promotes S phase entry. Cancer Lett 2001, 162:213-221.

14. Kociok N, Esser P, Unfried K, Parker F, Schraermeyer U, Grisanti S, Toque B, Heimann K: Upregulation of the RAS-GTPase activating protein (GAP)binding protein (G3BP) in proliferating RPE cells. J Cell Biochem 1999, 74:194-201.

15. Barnes CJ, Li F, Mandal M, Yang Z, Sahin AA, Kumar R: Heregulin induces expression, ATPase activity, and nuclear localization of G3BP, a Ras signaling component, in human breast tumors. Cancer Res 2002, 62:1251-1255

16. French J, Stirling R, Walsh M, Kennedy HD: The expression of Ras-GTPase activating protein $\mathrm{SH} 3$ domain-binding proteins, G3BPs, in human breast cancers. Histochem J 2002, 34:223-231.

17. Schneider C, King RM, Philipson L: Genes specifically expressed at growth arrest of mammalian cells. Cell 1988, 54:787-793.

18. Manfioletti G, Ruaro ME, Del Sal G, Philipson L, Schneider C: A growth arrest-specific (gas) gene codes for a membrane protein. Mol Cell Biol 1990, 10:2924-2930.

19. Zhang H, Zhang S, He H, Zhao W, Chen J, Shao RG: GAP161 targets and downregulates G3BP to suppress cell growth and potentiate cisplaitinmediated cytotoxicity to colon carcinoma HCT116 cells. Cancer Sci 2012, 103:1848-1856.

20. Irvine K, Stirling R, Hume D, Kennedy D: Rasputin, more promiscuous than ever: a review of G3BP. Int J Dev Biol 2004, 48:1065-1077.

21. Tourriere H, Chebli K, Zekri L, Courselaud B, Blanchard JM, Bertrand E, Tazi J: The RasGAP-associated endoribonuclease G3BP assembles stress granules. J Cell Biol 2003, 160:823-831.

22. Matsuki H, Takahashi M, Higuchi M, Makokha GN, Oie M, Fujii M: Both G3BP1 and G3BP2 contribute to stress granule formation. Genes Cells 2013, 18:135-146.

23. Kobayashi T, Winslow S, Sunesson L, Hellman U, Larsson C: PKCalpha binds G3BP2 and regulates stress granule formation following cellular stress. PLoS One 2012, 7:e35820.

24. Snipes GJ, Suter U, Welcher AA, Shooter EM: Characterization of a nove peripheral nervous system myelin protein (PMP-22/SR13). J Cell Biol 1992, 117:225-238.

25. Spreyer P, Kuhn G, Hanemann CO, Gillen C, Schaal H, Kuhn R, Lemke G, Muller HW: Axon-regulated expression of a Schwann cell transcript that is homologous to a 'growth arrest-specific' gene. EMBO J 1991, 10:3661-3668.

26. Muller HW: Tetraspan myelin protein PMP22 and demyelinating peripheral neuropathies: new facts and hypotheses. Glia 2000, 29:182-185.

27. Naef $R$, Suter $U$ : Impaired intracellular trafficking is a common disease mechanism of PMP22 point mutations in peripheral neuropathies. Neurobiol Dis 1999, 6:1-14.

28. Suter U, Nave KA: Transgenic mouse models of CMT1A and HNPP. Ann N Y Acad Sci 1999, 883:247-253.

29. Huhne K, Park O, Liehr T, Rautenstrauss B: Expression analysis of the PMP22 gene in glioma and osteogenic sarcoma cell lines. J Neurosci Res 1999, 58:624-631.

30. Li J, Kleeff J, Esposito I, Kayed H, Felix K, Giese T, Buchler MW, Friess H: Expression analysis of PMP22/Gas3 in premalignant and malignant pancreatic lesions. J Histochem Cytochem 2005, 53:885-893.

31. Tong D, Heinze G, Pils D, Wolf A, Singer CF, Concin N, Hofstetter G, Schiebel I, Rudas M, Zeillinger R: Gene expression of PMP22 is an independent prognostic factor for disease-free and overall survival in breast cancer patients. BMC Cancer 2010, 10:682 
32. Taylor V, Welcher AA, Program AE, Suter U: Epithelial membrane protein-1, peripheral myelin protein 22, and lens membrane protein 20 define a novel gene family. J Biol Chem 1995, 270:28824-28833.

33. van Dartel M, Hulsebos TJ: Characterization of PMP22 expression in osteosarcoma. Cancer Genet Cytogenet 2004, 152:113-118.

34. Roux KJ, Amici SA, Fletcher BS, Notterpek L: Modulation of epithelial morphology, monolayer permeability, and cell migration by growth arrest specific 3/peripheral myelin protein 22. Mol Biol Cell 2005, 16:1142-1151

35. Zoidl G, Blass-Kampmann S, D'Urso D, Schmalenbach C, Muller HW: Retroviral-mediated gene transfer of the peripheral myelin protein PMP22 in Schwann cells: modulation of cell growth. EMBO J 1995, 14:1122-1128

36. Verrier JD, Lau P, Hudson L, Murashov AK, Renne R, Notterpek L: Peripheral myelin protein 22 is regulated post-transcriptionally by miRNA-29a. Glia 2009, 57:1265-1279.

37. Du P, Kibbe WA, Lin SM: lumi: a pipeline for processing Illumina microarray. Bioinformatics 2008, 24:1547-1548.

doi:10.1186/1476-4598-12-156

Cite this article as: Winslow et al:: Regulation of PMP22 mRNA by G3BP1

affects cell proliferation in breast cancer cells. Molecular Cancer

2013 12:156.

\section{Submit your next manuscript to BioMed Central and take full advantage of:}

- Convenient online submission

- Thorough peer review

- No space constraints or color figure charges

- Immediate publication on acceptance

- Inclusion in PubMed, CAS, Scopus and Google Scholar

- Research which is freely available for redistribution 